\title{
A cross-country comparison of the prevalence of exposure to tobacco advertisements among adolescents aged 13-15 years in 20 low and middle income countries
}

\author{
Israel T Agaku ${ }^{1 *}$, Akinyele O Adisa ${ }^{2}$, Akindayo O Akinyamoju ${ }^{2}$ and Samuel O Agboola ${ }^{3}$
}

\begin{abstract}
Background: This study assessed the prevalence and influence of exposure to pro-tobacco advertisements among adolescents in 20 low and middle income countries (LMICs).

Methods: The 2007-2008 Global Youth Tobacco Survey was analyzed for students aged 13-15 years in 20 LMICs. Overall and sex-specific prevalence of exposure to tobacco advertisements in several media, as well as the prevalence of smoking susceptibility (i.e., the lack of a firm commitment among never smokers not to smoke in the future or if offered a cigarette by a friend) were assessed. The variability of the point estimates was assessed using 95\% confidence intervals (CI). Logistic regression was used to assess the effect of exposure to multiple (i.e., $\geq 2$ ) pro-tobacco advertisements on current smoking, adjusting for age and sex $(P<0.05)$. Data were weighted and analyzed with Stata version 11.

Results: Overall country-specific prevalence for different advertisement sources ranged as follows: movies/videos (78.4\% in Lesotho to $97.8 \%$ in Belize); television programs (48.7\% in Togo to $91.7 \%$ in the Philippines); newspapers/ magazines (29.5\% in Togo to $89.7 \%$ in the Philippines); and outdoor community events (30.6\% in Rwanda to $79.4 \%$ in the Philippines). The overall proportion of never smokers who were susceptible to cigarette smoking ranged from 3.7\% in Sri Lanka to $70.1 \%$ in Kyrgyzstan. Exposure to $\geq 2$ sources of pro-tobacco advertisements was associated with significantly increased odds of cigarette smoking among adolescents in several countries including South Africa (adjusted odds ratio, $a \mathrm{OR}=4.11 ; 95 \% \mathrm{Cl}: 2.26-7.47)$, Togo ( $a \mathrm{OR}=3.77 ; 95 \% \mathrm{Cl}: 1.27-11.21$ ), the Former Yugoslav Republic of Macedonia ( $a \mathrm{OR}=1.42 ; 95 \% \mathrm{Cl}: 1.01-1.99)$, Republic of Moldova ( $a \mathrm{OR}=1.53 ; 95 \% \mathrm{Cl}: 1.11-2.12$ ), Belize $(a \mathrm{OR}=13.95 ; 95 \% \mathrm{Cl}: 1.91-102.02)$, Panama $(a \mathrm{OR}=5.14 ; 95 \% \mathrm{Cl}: 2.37-11.14)$ and Mongolia $(a \mathrm{OR}=1.52$; 95\% Cl:1.19-1.94).

Conclusion: Prevalence of exposure to various pro-tobacco advertisements was high among adolescents in the LMICs surveyed. Enhanced and sustained national efforts are needed to reduce exposure to all forms of tobacco advertising and promotional activities.
\end{abstract}

Keywords: Advertisements, Tobacco promotion, Susceptibility, Smoking, Adolescents, Cigarette, Addiction, Developing countries

\footnotetext{
* Correspondence: iagaku@post.harvard.edu

${ }^{1}$ Africa Tobacco Control Regional Initiative, Plot 397B, George Crescent,

Agbalajobi Estate, Off Wempco Road, Lagos, Ogba-lkeja, Nigeria

Full list of author information is available at the end of the article
} 


\section{Introduction}

As declines in cigarette smoking occur in developed nations in recent years, the tobacco industry has increasingly targeted low and middle income countries (LMICs) in order to increase sales and profits [1]. During 2005 and 2010, the volume of sales of cigarettes declined by $12 \%$ in Western Europe and 20\% in North America, while increasing by $17 \%$ in the Middle East region and North Africa combined [2]. Along with Asia-Pacific, the Middle East and Africa have continued to see growths in cigarette volume sales at a rate higher than the rest of the world [2-4]. The Euromonitor International- a global market research report- has projected a $7 \%$ growth in the number of smokers in the Middle East and Africa between 2010 and 2015- the fastest growth rate of smokers in the world [5]. Changes in demographics (favoring a growth in the young adult population), coupled with relatively lax tobacco control policies and lower levels of health awareness, have all contributed to the boost in volume sales of cigarettes observed in LMICs in recent years [2]. In addition, the tobacco industry has established a strong presence in several LMICs, as indicated by the construction of multi-million dollar tobacco plants in several countries including Nigeria, Senegal, and South-Africa, among others [6-8]. More so, political unrest in several LMICs including Egypt, Libya, Tunisia, Yemen, and Bahrain, may have contributed to a boost in illicit trade of tobacco products [2].

The susceptibility of youths to social influences to use tobacco products has made them an important target of the tobacco industry [9]. Research has suggested that certain tobacco companies developed a global brand through strategic advertising and marketing plans that targeted youths by highlighting themes designed to appeal to young people such as independence, hedonism, freedom, and comfort [10]. The industry has also used corporate social responsibility tactics to improve its corporate image [11], and promote tobacco products among youths. In addition, the increased expenditures on internet advertising of tobacco products by major cigarette manufacturers in recent years [12] may likely have an influence on adolescents in LMICs due to the global reach of such internet marketing activities. Such promotion of tobacco products to youths undermines national and international efforts to protect children and youths from the dangers of tobacco use [13]. The United States' Surgeon General's report has concluded that such tobacco promotional activities have a causal effect on smoking initiation among young people [9].

Despite these developments, only very little information exists on the reach and effect of pro-tobacco advertising among youths in developing countries [14]. To fill this gap in knowledge, this study assessed the prevalence and correlates of pro-tobacco advertisements among adolescents aged $13-15$ years in 20 LMICs, as well as the prevalence of smoking susceptibility among adolescents that had never smoked cigarettes in these countries, using data from the Global Youth Tobacco Survey.

\section{Methods}

\section{Data source}

\section{Global youth tobacco survey (GYTS)}

The GYTS is a school-based survey that was initiated by the World Health Organization (WHO); the United States Centers for Disease Control and Prevention, and the Canadian Public Health Association. The survey collects tobacco use data at four-year intervals from students aged 13-15 years using a two stage cluster sample. Schools are selected with probability proportional to school enrolment size during the first stage, and then classes within participating schools are selected as a systematic equal probability sample with a random start during the second stage. All students in the selected classes are eligible to participate in the survey. The GYTS survey methodology has been described in detail elsewhere [15].

\section{Sample}

For this analysis, we excluded all sub-national data (i.e., provincial, city or state level data) to allow direct comparisons of country-level data. Also, all countries whose most recent national GYTS data was collected prior to 2007 were excluded to allow comparisons of more recent data. In total, 2007-2008 data was analyzed for 20 LMICs (defined as countries with gross national income per capita $\leq \$ 12,475$ as of 2011) [16]. This categorization included low income, lower-middle income, and upper middle income countries [16].

The 20 countries for which data was available for the outcome measures of interest are presented in this study by geographic region based on the six WHO regions. These included: African region $(n=6$ countries: Botswana; Lesotho; Rwanda; Seychelles; South Africa and Togo); Eastern Mediterranean region ( $n=3$ countries: Islamic Republic of Iran; Tunisia and Yemen); Europe ( $n=5$ countries: Kyrgyzstan; the Former Yugoslav Republic of Macedonia; Republic of Moldova; Montenegro; and Serbia); Region of the Americas ( $n=2$ countries: Belize; and Panama); South-East Asia ( $n=2$ countries: Myanmar and Sri Lanka) and Western Pacific region ( $n=2$ countries: Mongolia; and Philippines).

\section{Measures}

Questionnaires were standardized and selected question items were similarly worded for all countries, thus allowing for direct cross-country comparisons. 


\section{Current cigarette smoking}

Current cigarette smoking was defined as a report by a student that they had smoked cigarettes on $\geq 1$ day during the past 30 days.

\section{Smoking susceptibility}

Respondents who had never experimented with cigarette smoking, even one or two puffs, were defined as "never smokers." Never smokers who were susceptible to smoking were defined as those who responded in any way other than "Definitely not" to the following two questions: "at any time during the next 12 months, do you think you will smoke a cigarette?" or "if one of your best friends offered you a cigarette, would you smoke it?"

\section{Exposure to smoking incidents in movies/videos}

Exposure to smoking incidents in movies/videos was assessed using the question: "When you watch TV, videos, or movies, how often do you see actors smoking?" Respondents who answered "I never watch TV, videos, or movies" were excluded from the analysis, whereas those who answered "a lot" or "sometimes" were categorized as being exposed to smoking incidents in movies/ videos. Respondents who answered "never" were categorized as being non-exposed.

\section{Exposure to pro-tobacco advertisements on television programs}

Exposure to pro-tobacco advertisements on television programs was assessed using the question: "During the past 30 days (one month), when you watched sports events or other programs on TV how often did you see cigarette brand names?" Respondents who answered "I never watch TV" were excluded from the analysis, whereas those who answered "a lot" or "sometimes" were categorized as being exposed to pro-tobacco advertisements in television programs. Respondents who answered "never" were categorized as being non-exposed.

\section{Exposure to pro-tobacco advertisements in newspapers/ magazines}

Exposure to pro-tobacco advertisements in newspapers/ magazines was assessed using the question: "During the past 30 days (one month), how many advertisements or promotions for cigarettes have you seen in newspapers or magazines?" Respondents who answered "A lot" or "A few" were categorized as being exposed to pro-tobacco advertisements in newspapers/magazines whereas those who answered "None" were categorized as being non-exposed.

\section{Exposure to pro-tobacco advertisements at outdoor community events}

Exposure to pro-tobacco advertisements at outdoor community events was assessed using the question: "when you go to sports events, musical concerts, community events, or cultural festivals, how often do you see advertisements for cigarettes?" Respondents who answered "I never attend sports, musical concerts, community events, or cultural festival" were excluded from the analysis, whereas those who answered "a lot" or "sometimes" were categorized as being exposed to protobacco advertisements at outdoor community events. Respondents who answered "never" were categorized as being non-exposed.

\section{Socio-demographic characteristics}

Socio-demographic characteristics assessed included age (13, 14 or 15 years); and sex (boy or girl).

\section{Statistical analysis}

The target population of the GYTS in each country was students aged 13-15 years. Hence, we restricted all analyses to students within those ages for all 20 countries to maintain an identical population regardless of country-specific school/class grading system. This enhanced comparability of national estimates.

Data for each country were weighted to reflect the initial probabilities of selection and non-response patterns, and to post-stratify the data to known sampling frame characteristics. The prevalence of exposure to various pro-tobacco advertisements, and the proportion of never smokers who were susceptible to cigarette smoking, were assessed overall for each country and also stratified by sex; $95 \%$ confidence intervals (CI) were calculated to account for the complex survey design. Pair-wise comparison of country estimates was done using a 2 sample $t$-test. All tests were 2-tailed and the level of statistical significance was set at $P<0.05$.

In addition, for each country, the cumulative number of pro-tobacco advertisements respondents reported being exposed to was added for each student (range: 0 to 4). This was then dichotomized as exposure to $\leq 1$ source of pro-tobacco advertisement vs. exposure to multiple sources of pro-tobacco advertisements (2 to 4). Multivariate logistic regression was used to assess the effect of exposure to multiple pro-tobacco advertisements on current smoking, adjusting for age and sex. All analyses were performed with Stata version 11 (Stata Corp. 2009. Stata Statistical Software: College Station, TX).

\section{Results}

Total sample sizes for all students ranged from $n=1219$ in Yemen, to $n=8602$ in South Africa. The proportion of 13-15 year olds among the total number of students sampled ranged from $30.2 \%$ in Rwanda to $80.3 \%$ in Panama. The proportion of boys aged 13-15 years ranged from $37.5 \%$ in Lesotho to $62.5 \%$ in Yemen. 
Table 1 Characteristics of National Surveys of adolescents in $\mathbf{2 0}$ low and middle income countries, 2007-2008

\begin{tabular}{|c|c|c|c|c|c|c|}
\hline Region/Country & $\begin{array}{r}\text { Survey } \\
\text { year }\end{array}$ & $\begin{array}{r}\text { Total sample } \\
\text { size, } n\end{array}$ & $\begin{array}{r}\text { Overall response } \\
\text { rate(\%) }\end{array}$ & $\begin{array}{r}\% \text { of all respondents } \\
\text { aged } 13-15 \text { years }\end{array}$ & $\begin{array}{r}\% \text { composition: } \\
\text { boys* }\end{array}$ & $\begin{array}{r}\text { \% composition: } \\
\text { girls* }\end{array}$ \\
\hline \multicolumn{7}{|l|}{ African Region } \\
\hline Botswana & 2008 & 2207 & 96.0 & 72.8 & 41.6 & 58.4 \\
\hline Lesotho & 2008 & 3426 & 83.2 & 49.8 & 37.5 & 62.5 \\
\hline Rwanda & 2008 & 2284 & 91.8 & 30.2 & 47.2 & 52.8 \\
\hline Seychelles & 2007 & 1508 & 86.0 & 57.0 & 49.4 & 50.6 \\
\hline South Africa & 2008 & 8602 & 77.9 & 46.0 & 42.3 & 57.7 \\
\hline Togo & 2007 & 4262 & 89.9 & 46.9 & 59.6 & 40.4 \\
\hline \multicolumn{7}{|l|}{$\begin{array}{l}\text { Eastern Mediterranean } \\
\text { region }\end{array}$} \\
\hline Islamic Republic of Iran & 2007 & 1996 & 85.9 & 66.6 & 52.1 & 47.9 \\
\hline Tunisia & 2007 & 2155 & 92.4 & 70.3 & 50.1 & 49.9 \\
\hline Yemen & 2008 & 1219 & 83.5 & 52.7 & 62.5 & 37.5 \\
\hline \multicolumn{7}{|l|}{ Europe } \\
\hline Kyrgyzstan & 2008 & 4038 & 93.2 & 74.4 & 47.4 & 52.6 \\
\hline FYR Macedonia & 2008 & 5824 & 90.1 & 73.5 & 50.9 & 49.1 \\
\hline Republic of Moldova & 2008 & 4703 & 84.3 & 75.1 & 44.4 & 55.6 \\
\hline Montenegro & 2008 & 5723 & 92.9 & 58.5 & 48.1 & 51.9 \\
\hline Serbia & 2008 & 4727 & 89.4 & 67.4 & 45.4 & 54.6 \\
\hline \multicolumn{7}{|l|}{ Region of the Americas } \\
\hline Belize & 2008 & 1751 & 93.9 & 61.1 & 47.0 & 53.0 \\
\hline Panama & 2008 & 3543 & 80.0 & 80.3 & 47.1 & 52.9 \\
\hline \multicolumn{7}{|l|}{ South-East Asia } \\
\hline Myanmar & 2007 & 3118 & 95.2 & 69.5 & 48.9 & 51.2 \\
\hline Sri Lanka & 2007 & 1764 & 85.0 & 79.8 & 50.2 & 49.8 \\
\hline \multicolumn{7}{|l|}{ Western Pacific } \\
\hline Mongolia & 2007 & 1831 & 51.9 & 77.8 & 46.6 & 53.4 \\
\hline Philippines & 2007 & 5919 & 80.9 & 56.4 & 44.2 & 55.8 \\
\hline
\end{tabular}

Note: Sample sizes " $\mathrm{n}$ " are unweighted while percentages are weighted. FYR Macedonia=the Former Yugoslav Republic of Macedonia.

*Among respondents aged $13-15$ years.

Overall response rates ranged from 51.9\% (Mongolia) to 96.0\% (Botswana) (Table 1).

Movies/videos constituted the most common source of exposure to pro-tobacco influence among adolescents aged 13-15 years. The prevalence of exposure to smoking incidents in movies/videos ranged from 78.4\% (Lesotho) to $97.8 \%$ (Belize) (Table 2). Prevalence of exposure to pro-tobacco advertisements on television programs ranged from $48.7 \%$ in Togo to $91.7 \%$ in the Philippines. Prevalence estimates of exposure to protobacco advertisements in newspapers/magazines were generally lower compared to the electronic (i.e., movies/ videos and television programs) routes of exposure, and ranged from $29.5 \%$ (Togo) to $89.7 \%$ (Philippines). Prevalence of exposure to pro-tobacco advertisements at outdoor community events ranged from 30.6\% (Rwanda) to $79.4 \%$ (Sri Lanka). There was no significant difference by sex for all types of exposure to pro-tobacco advertisements in all countries surveyed.

The proportion of never smokers who were susceptible to cigarette smoking ranged from $3.7 \%$ in Sri Lanka to $70.1 \%$ in Kyrgyzstan (Table 3). The proportion of boys that were susceptible to cigarette smoking was significantly higher than the proportion of susceptible girls in the following countries: Botswana, Tunisia, Panama and Myanmar. In all other countries however, there was no significant gender difference in the prevalence of smoking susceptibility among never smokers.

After adjusting for age and sex, exposure to $\geq 2$ sources of pro-tobacco advertisements was associated with significantly increased odds of cigarette smoking among adolescents in several countries including South Africa (adjusted odds ratio, $a \mathrm{OR}=4.11 ; 95 \%$ CI:2.26-7.47); Togo $(a \mathrm{OR}=3.77 ; 95 \% \mathrm{CI}: 1.27-11.21)$; the Former 
Table 2 Prevalence of exposure to pro-tobacco advertisements in movies/videos, television programs, newspapers/magazines and at outdoor community events among students aged 13-15 years in 20 low and middle income countries, 2007-2008

\begin{tabular}{|c|c|c|c|c|c|c|c|c|c|c|c|c|}
\hline \multirow[t]{3}{*}{ Region/Country } & \multicolumn{3}{|c|}{ Movies/Videos } & \multicolumn{3}{|c|}{ Television programs } & \multicolumn{3}{|c|}{ Newspapers/Magazines } & \multicolumn{3}{|c|}{ Outdoor community events } \\
\hline & Overall & Boys & Girls & Overall & Boys & Girls & Overall & Boys & Girls & Overall & Boys & Girls \\
\hline & $\%(95 \% \mathrm{Cl})$ & $\%(95 \% \mathrm{Cl})$ & $\%(95 \% \mathrm{Cl})$ & $\%(95 \% \mathrm{Cl})$ & $\%(95 \% \mathrm{Cl})$ & $\%(95 \% \mathrm{Cl})$ & $\%(95 \% \mathrm{Cl})$ & $\%(95 \% \mathrm{Cl})$ & $\%(95 \% \mathrm{Cl})$ & $\%(95 \% \mathrm{Cl})$ & $\%(95 \% \mathrm{Cl})$ & $\%(95 \% \mathrm{Cl})$ \\
\hline \multicolumn{13}{|l|}{ African Region } \\
\hline \multirow[t]{2}{*}{ Botswana } & 90.7 & 91.7 & 90.0 & 68.0 & 65.3 & 70.4 & 48.4 & 47.4 & 49.2 & 55.8 & 56.8 & 54.3 \\
\hline & $(88.7-92.7)$ & $(88.4-94.9)$ & $(87.7-92.3)$ & $(65.6-70.4)$ & $(61.9-68.7)$ & $(66.9-74.0)$ & $(45.1-51.8)$ & $(43.4-51.5)$ & $(45.3-53.1)$ & $(52.8-58.9)$ & $(52.6-61.0)$ & $(50.4-58.2)$ \\
\hline \multirow[t]{2}{*}{ Lesotho } & 78.4 & 74.7 & 80.3 & 70.8 & 71.0 & 69.5 & 60.2 & 59.9 & 60.3 & 62.4 & 58.1 & 65.0 \\
\hline & $(70.1-86.8)$ & $(62.5-86.9)$ & (73.8-86.9) & $(65.8-75.8)$ & $(64.5-77.6)$ & $(63.8-75.1)$ & $(55.9-64.6)$ & $(53.4-66.5)$ & $(54.0-66.6)$ & $(54.2-70.5)$ & $(49.9-66.2)$ & $(56.2-73.8)$ \\
\hline \multirow[t]{2}{*}{ Rwanda } & 86.4 & 88.8 & 83.9 & 79.0 & 83.1 & 74.7 & 31.1 & 29.2 & 31.9 & 30.6 & 31.2 & 29.4 \\
\hline & $(81.4-91.5)$ & $(83.8-93.9)$ & $(76.6-91.3)$ & $(73.4-84.6)$ & $(78.1-88.1)$ & $(67.2-82.2)$ & $(26.2-35.9)$ & $(24.5-33.9)$ & $(23.5-40.3)$ & $(25.9-35.3)$ & $(25.1-37.3)$ & $(21.1-37.7)$ \\
\hline \multirow[t]{2}{*}{ Seychelles } & 96.1 & 94.8 & 97.2 & 68.1 & 65.1 & 71.3 & 49.4 & 47.5 & 50.8 & 63.6 & 57.5 & 69.6 \\
\hline & $(94.0-98.1)$ & $(91.4-98.2)$ & $(95.5-98.9)$ & $(63.9-72.2)$ & $(59.3-70.9)$ & (64.8-77.8) & $(43.3-55.4)$ & $(40.9-54.0)$ & $(42.4-59.3)$ & (58.8-68.3) & $(50.8-64.1)$ & $(64.6-74.7)$ \\
\hline \multirow[t]{2}{*}{ South Africa } & 92.7 & 91.9 & 93.2 & 77.2 & 78.3 & 76.5 & 66.8 & 67.7 & 66.1 & 59.7 & 60.1 & 59.4 \\
\hline & $(90.8-94.5)$ & $(89.3-94.6)$ & $(91.3-95.2)$ & $(74.9-79.5)$ & $(75.5-81.0)$ & $(73.5-79.5)$ & $(63.6-70.1)$ & $(64.1-71.4)$ & $(62.2-70.0)$ & $(57.0-62.3)$ & $(56.6-63.7)$ & $(56.3-62.6)$ \\
\hline \multirow[t]{2}{*}{ Togo } & 80.0 & 79.6 & 80.5 & 48.7 & 46.8 & 50.8 & 29.5 & 29.2 & 29.5 & 44.3 & 43.7 & 44.7 \\
\hline & $(74.0-86.0)$ & $(72.7-86.4)$ & $(74.5-86.4)$ & $(40.6-56.8)$ & $(36.7-56.8)$ & $(43.5-58.1)$ & $(23.0-35.9)$ & $(21.7-36.7)$ & (23.6-35.3) & $(34.3-54.2)$ & (33.5-53.9) & (33.4-55.9) \\
\hline \multicolumn{13}{|c|}{ Eastern Mediterranean region } \\
\hline \multirow[t]{2}{*}{ Islamic Republic of Iran } & 94.7 & 93.2 & 96.3 & 64.8 & 64.6 & 64.8 & 48.2 & 48.0 & 48.4 & 59.8 & 60.4 & 58.6 \\
\hline & $(93.2-96.3)$ & $(91.5-94.9)$ & $(94.3-98.4)$ & $(58.6-71.0)$ & $(56.4-72.9)$ & $(57.3-72.4)$ & $(43.0-53.3)$ & $(39.3-56.7)$ & $(41.3-55.5)$ & $(53.2-66.3)$ & $(50.8-70.1)$ & $(49.5-67.6)$ \\
\hline \multirow[t]{2}{*}{ Tunisia } & 97.6 & 96.8 & 98.3 & 68.3 & 69.5 & 67.0 & 58.7 & 58.4 & 59.0 & 64.4 & 66.8 & 61.4 \\
\hline & $(96.6-98.6)$ & $(95.6-98.0)$ & $(97.3-99.3)$ & $(65.1-71.5)$ & $(65.7-73.3)$ & $(62.7-71.4)$ & $(55.0-62.5)$ & $(53.9-62.9)$ & $(53.5-64.5)$ & $(60.3-68.5)$ & $(63.3-70.4)$ & $(55.6-67.2)$ \\
\hline \multirow[t]{2}{*}{ Yemen } & 90.4 & 90.1 & 91.4 & 77.6 & 79.4 & 73.4 & 62.3 & 64.4 & 57.9 & 62.6 & 61.3 & 64.1 \\
\hline & $(85.8-95.0)$ & $(83.7-96.6)$ & $(85.0-97.8)$ & $(72.1-83.1)$ & $(70.8-88.0)$ & $(61.3-85.6)$ & $(53.2-71.3)$ & $(53.3-75.6)$ & $(48.8-67.0)$ & $(52.7-72.4)$ & $(47.6-74.9)$ & $(51.5-76.7)$ \\
\hline \multicolumn{13}{|l|}{ Europe } \\
\hline \multirow[t]{2}{*}{ Kyrgyzstan } & 93.4 & 92.4 & 94.4 & 72.7 & 70.7 & 74.5 & 55.2 & 58.9 & 51.9 & 56.7 & 53.1 & 59.8 \\
\hline & $(91.0-95.9)$ & $(88.3-96.5)$ & $(92.4-96.4)$ & $(70.4-75.0)$ & $(67.1-74.3)$ & $(70.7-78.4)$ & $(51.8-58.7)$ & $(54.9-62.9)$ & $(47.1-56.8)$ & $(51.5-61.8)$ & $(46.7-59.5)$ & $(54.8-64.8)$ \\
\hline \multirow[t]{2}{*}{ FYR Macedonia } & 92.9 & 92.9 & 92.9 & 71.0 & 70.8 & 71.0 & 65.2 & 63.4 & 67.0 & 57.9 & 58.2 & 57.7 \\
\hline & $(91.3-94.5)$ & $(91.1-94.7)$ & $(90.8-94.9)$ & $(68.9-73.1)$ & $(67.8-73.8)$ & $(68.4-73.7)$ & $(63.2-67.2)$ & $(61.0-65.9)$ & $(63.9-70.1)$ & $(55.9-60.0)$ & $(55.8-60.5)$ & $(54.4-61.0)$ \\
\hline \multirow[t]{2}{*}{ Republic of Moldova } & 94.1 & 94.0 & 94.4 & 66.6 & 68.4 & 64.9 & 58.1 & 58.6 & 57.7 & 43.6 & 45.2 & 41.9 \\
\hline & $(93.0-95.3)$ & $(92.6-95.5)$ & (93.0-95.9) & $(64.3-69.0)$ & $(65.5-71.2)$ & $(61.3-68.5)$ & $(55.6-60.6)$ & $(54.1-63.1)$ & $(53.8-61.6)$ & $(40.2-47.1)$ & $(41.1-49.2)$ & $(37.9-46.0)$ \\
\hline \multirow[t]{2}{*}{ Montenegro } & 96.7 & 96.1 & 97.4 & 66.5 & 67.7 & 65.5 & 58.2 & 58.3 & 58.1 & 43.6 & 43.5 & 43.2 \\
\hline & $(96.0-97.5)$ & $(95.3-96.9)$ & (96.3-98.6) & $(64.5-68.6)$ & $(64.3-71.0)$ & $(62.3-68.6)$ & $(55.2-61.1)$ & $(54.4-62.2)$ & $(53.9-62.3)$ & $(40.5-46.7)$ & $(39.9-47.1)$ & (39.0-47.5) \\
\hline
\end{tabular}


Table 2 Prevalence of exposure to pro-tobacco advertisements in movies/videos, television programs, newspapers/magazines and at outdoor community events among students aged 13-15 years in 20 low and middle income countries, 2007-2008 (Continued)

\begin{tabular}{|c|c|c|c|c|c|c|c|c|c|c|c|c|}
\hline \multirow[t]{2}{*}{ Serbia } & 97.3 & 96.3 & 98.4 & 73.8 & 75.3 & 73.0 & 63.8 & 65.2 & 62.8 & 53.9 & 54.0 & 53.2 \\
\hline & $(96.4-98.2)$ & $(94.5-98.0)$ & $(97.7-99.2)$ & $(71.6-76.1)$ & $(72.1-78.5)$ & $(69.7-76.2)$ & $(61.5-66.2)$ & $(61.9-68.5)$ & $(59.8-65.7)$ & $(51.2-56.6)$ & $(50.1-58.0)$ & $(49.7-56.6)$ \\
\hline \multicolumn{13}{|c|}{ Region of the Americas } \\
\hline \multirow[t]{2}{*}{ Belize } & 97.8 & 97.9 & 97.7 & 77.1 & 78.0 & 76.2 & 58.3 & 59.7 & 56.7 & 64.0 & 64.0 & 64.1 \\
\hline & $(97.1-98.4)$ & $(96.9-98.8)$ & $(96.6-98.7)$ & $(72.8-81.5)$ & $(73.2-82.8)$ & $(70.6-81.7)$ & $(53.5-63.1)$ & $(53.8-65.6)$ & $(51.5-62.0)$ & $(59.0-69.0)$ & $(58.0-70.1)$ & $(58.1-70.0)$ \\
\hline \multirow[t]{2}{*}{ Panama } & 87.1 & 87.6 & 86.6 & 57.8 & 59.5 & 56.2 & 56.7 & 59.9 & 54.3 & $--^{*}$ & $--^{*}$ & $--{ }^{*}$ \\
\hline & $(84.9-89.3)$ & $(84.8-90.4)$ & $(84.1-89.2)$ & $(55.7-59.8)$ & $(55.6-63.4)$ & $(52.8-59.6)$ & $(54.3-59.2)$ & $(56.6-63.3)$ & $(50.2-58.3)$ & & & \\
\hline \multicolumn{13}{|c|}{ South-East Asia } \\
\hline \multirow[t]{2}{*}{ Myanmar } & 94.3 & 94.4 & 94.4 & 65.1 & 64.3 & 66.0 & 78.7 & 78.5 & 78.9 & 67.2 & 68.8 & 65.2 \\
\hline & $(92.4-96.2)$ & $(91.8-96.9)$ & $(91.5-97.2)$ & $(61.6-68.6)$ & $(60.7-68.0)$ & $(61.7-70.4)$ & (74.9-82.4) & (74.0-82.9) & (74.1-83.6) & (63.0-71.4) & $(63.5-74.2)$ & $(60.0-70.5)$ \\
\hline \multirow[t]{2}{*}{ Sri Lanka } & 93.6 & 92.7 & 94.5 & 72.8 & 74.5 & 71.2 & 68.4 & 68.8 & 68.0 & 79.0 & 78.3 & 79.6 \\
\hline & (91.9-95.4) & (89.6-95.7) & $(92.8-96.3)$ & $(68.7-76.9)$ & $(69.4-79.7)$ & $(66.7-75.7)$ & $(64.6-72.3)$ & $(62.4-75.1)$ & $(63.2-72.8)$ & (75.5-82.5) & (73.6-82.9) & $(75.1-84.2)$ \\
\hline \multicolumn{13}{|c|}{ Western Pacific } \\
\hline \multirow[t]{2}{*}{ Mongolia } & 80.9 & 81 & 80.7 & 54.6 & 57.4 & 51.5 & 40.8 & 42.1 & 39.2 & 48.9 & 47.0 & 50.1 \\
\hline & $(74.1-87.8)$ & (74.3-87.6) & $(73.1-88.2)$ & $(46.2-63)$ & $(47.0-67.8)$ & $(44.2-58.8)$ & $(35.5-46.1)$ & $(36.4-47.8)$ & $(33.1-45.4)$ & $(39.6-58.3)$ & $(34.6-59.5)$ & $(42.9-57.4)$ \\
\hline \multirow[t]{2}{*}{ Philippines } & 93.1 & 94 & 92.3 & 91.7 & 90.5 & 92.8 & 89.7 & 93.5 & 86.0 & 79.4 & 81.1 & 78.0 \\
\hline & $(92.2-93.9)$ & $(92.5-95.6)$ & $(91-93.6)$ & $(90.1-93.3)$ & $(88.1-92.9)$ & $(91.1-94.4)$ & $(83.2-96.3)$ & (83.9-103) & $(77.2-94.8)$ & $(77.5-81.3)$ & $(78.1-84.1)$ & $(75.4-80.5)$ \\
\hline
\end{tabular}

Note: $\mathrm{Cl}$, confidence interval; FYR Macedonia, the Former Yugoslav Republic of Macedonia.

* Outcome measure not calculated because of non-availability of data (i.e., question was not asked). 
Table 3 Proportion of never smokers aged 13-15 years in 20 low and middle income countries who were susceptible to cigarette smoking, 2007-2008

\begin{tabular}{|c|c|c|c|}
\hline Region/Country & $\begin{array}{l}\text { Overall proportion of never smokers } \\
\text { who were susceptible to cigarette } \\
\text { smoking, } \%(95 \% \mathrm{Cl})\end{array}$ & $\begin{array}{l}\text { Proportion of male never smokers } \\
\text { who were susceptible to cigarette } \\
\text { smoking, } \%(95 \% \mathrm{Cl})\end{array}$ & $\begin{array}{l}\text { Proportion of female never smokers } \\
\text { who were susceptible to cigarette } \\
\text { smoking, } \%(95 \% \mathrm{Cl})\end{array}$ \\
\hline \multicolumn{4}{|l|}{ African Region } \\
\hline Botswana & $27.1(22.5-31.7)$ & $33.3(27.9-38.7)$ & $22.5(17.6-27.4)$ \\
\hline Lesotho & $33.7(27.0-40.3)$ & $33.7(24.9-42.5)$ & $33.1(26.0-40.1)$ \\
\hline Madagascar & $12.5(8.1-17.0)$ & $12.3(6.1-18.5)$ & $12.5(7.0-18.1)$ \\
\hline Rwanda & $10.0(7.1-12.9)$ & $12(8.1-15.9)$ & $7.8(4.8-10.8)$ \\
\hline Seychelles & $15.4(11.7-19.0)$ & $14.4(8.6-20.2)$ & $16.2(12.0-20.4)$ \\
\hline South Africa & $15.4(13.2-17.6)$ & $17.4(14.3-20.5)$ & $14.3(11.6-16.9)$ \\
\hline Togo & $9.2(6.5-12.0)$ & $9.7(6.1-13.3)$ & $8.5(5.8-11.2)$ \\
\hline \multicolumn{4}{|l|}{$\begin{array}{l}\text { Eastern Mediterranean } \\
\text { region }\end{array}$} \\
\hline Islamic Republic of Iran & $8.7(6.9-10.5)$ & $10.3(7.6-13.0)$ & $7.0(4.6-9.4)$ \\
\hline Tunisia & $19.9(16.2-23.7)$ & $26.7(21.6-31.8)$ & $15.5(11.3-19.8)$ \\
\hline Yemen & $24.1(18.8-29.4)$ & $22.1(13.4-30.9)$ & $27.4(20.5-34.2)$ \\
\hline \multicolumn{4}{|l|}{ Europe } \\
\hline Kyrgyzstan & $70.1(64.9-75.3)$ & $64.6(57.3-72.0)$ & $74.2(68.4-80.0)$ \\
\hline FYR Macedonia & $16.7(14.9-18.4)$ & $15.4(13.3-17.6)$ & $17.9(15.4-20.4)$ \\
\hline Republic of Moldova & $18.7(15.6-21.8)$ & $19.7(15.2-24.1)$ & $18.1(14.4-21.8)$ \\
\hline Montenegro & $16.0(14.0-18.1)$ & $15.7(12.0-19.5)$ & $16.5(13.7-19.2)$ \\
\hline Serbia & $19.0(16.7-21.3)$ & $16.2(12.5-20.0)$ & $20.9(17.9-23.8)$ \\
\hline \multicolumn{4}{|l|}{ Region of the Americas } \\
\hline Belize & $21.3(18.5-24.2)$ & $25.1(20.3-29.9)$ & $18.8(14.1-23.4)$ \\
\hline Panama & $10.0(8.7-11.3)$ & $12.3(10.5-14.1)$ & $8.3(6.3-10.2)$ \\
\hline \multicolumn{4}{|l|}{ South-East Asia } \\
\hline Myanmar & $11.4(9.3-13.6)$ & $15.9(12.1-19.6)$ & $8.1(5.9-10.3)$ \\
\hline Sri Lanka & $3.7(2.1-5.3)$ & $5.2(2.5-7.9)$ & $2.2(0.8-3.7)$ \\
\hline \multicolumn{4}{|l|}{ Western Pacific } \\
\hline Mongolia & $8.1(5.5-10.8)$ & $8.8(4.8-12.8)$ & $7.8(3.8-11.8)$ \\
\hline Philippines & $12.9(11.0-14.9)$ & $15.0(11.7-18.4)$ & $11.6(9.4-13.7)$ \\
\hline
\end{tabular}

Note: $C l$, confidence interval; FYR Macedonia, the Former Yugoslav Republic of Macedonia.

Yugoslav Republic of Macedonia $(a \mathrm{OR}=1.42 ; 95 \% \mathrm{CI}$ : 1.01-1.99); Republic of Moldova $(a \mathrm{OR}=1.53 ; 95 \% \mathrm{CI}$ : 1.11-2.12); Belize ( $a \mathrm{OR}=13.95 ; 95 \% \mathrm{CI}: 1.91-102.02)$; Panama $(a \mathrm{OR}=5.14 ; 95 \% \mathrm{CI}: 2.37-11.14)$; and Mongolia $(a \mathrm{OR}=1.52$; 95\% CI: 1.19-1.94) $($ Table 4).

\section{Discussion}

This study showed that the overall prevalence of exposure to pro-tobacco advertisements from several routes (including movies/videos, television programs, newspapers/magazines and outdoor community events) among adolescents aged 13-15 years was high in all the countries surveyed. Considering that all the 20 LMICs countries assessed in this study are parties to the WHO Framework Convention on Tobacco Control [17], which encourages parties to undertake a comprehensive ban of all tobacco advertising and promotion activities (Article 13) [13], these findings indicate that there is need for stronger implementation of domestic policies in individual countries to reduce the impact of pro-tobacco social influences.

Smoking by children and adolescents and the targeting of tobacco promotional activities to this demographic group is a fundamental issue in tobacco control. Our study showed that exposure to multiple sources of protobacco advertisements was associated with smoking behavior among adolescents in several LMICs. In addition, the prevalence of smoking susceptibility among never smokers was $>15 \%$ in 12 out of the 20 countries assessed. This underscores the need for policies to 
Table 4 Effect of exposure to multiple sources of tobacco advertisements on current smoking among adolescents aged 13-15 years in 20 low and middle income countries, 2007-2008

\begin{tabular}{|c|c|c|}
\hline Region/Country & $\begin{array}{l}\text { Unadjusted effect of } \\
\text { exposure to multiple } \\
\text { pro-tobacco } \\
\text { advertisements on } \\
\text { current cigarette } \\
\text { smoking, cOR }(95 \% \mathrm{Cl})\end{array}$ & $\begin{array}{l}\text { Adjusted effect of } \\
\text { exposure to multiple } \\
\text { pro-tobacco } \\
\text { advertisements on } \\
\text { current cigarette } \\
\text { smoking, aOR }(95 \% \mathrm{Cl})^{*}\end{array}$ \\
\hline \multicolumn{3}{|l|}{ African Region } \\
\hline Botswana & $1.14(0.73-1.80)$ & $1.39(0.80-2.42)$ \\
\hline Lesotho & $10.01(3.10-32.38)$ & $6.68(0.97-46.26)$ \\
\hline Madagascar & $2.89(1.05-7.96)$ & $2.82(0.70-11.42)$ \\
\hline Rwanda & $3.52(1.16-10.74)$ & $2.97(0.95-9.29)$ \\
\hline Seychelles & $1.40(0.87-2.26)$ & $1.63(0.98-2.71)$ \\
\hline South Africa & $2.61(1.59-4.28)$ & $4.11(2.26-7.47)^{\dagger}$ \\
\hline Togo & $2.88(1.51-5.48)$ & $3.77(1.27-11.21)^{\dagger}$ \\
\hline \multicolumn{3}{|l|}{$\begin{array}{l}\text { Eastern } \\
\text { Mediterranean } \\
\text { region }\end{array}$} \\
\hline $\begin{array}{l}\text { Islamic Republic of } \\
\text { Iran }\end{array}$ & $0.86(0.40-1.82)$ & $1.28(0.61-2.68)$ \\
\hline Tunisia & $1.31(0.70-2.46)$ & $2.12(0.87-5.15)$ \\
\hline Yemen & $1.47(0.88-2.45)$ & $1.70(0.74-3.90)$ \\
\hline \multicolumn{3}{|l|}{ Europe } \\
\hline Kyrgyzstan & $0.65(0.28-1.52)$ & $1.09(0.37-3.20)$ \\
\hline FYR Macedonia & $1.44(1.07-1.94)$ & $1.42(1.01-1.99)^{+}$ \\
\hline $\begin{array}{l}\text { Republic of } \\
\text { Moldova }\end{array}$ & $1.65(1.23-2.22)$ & $1.53(1.11-2.12)^{\dagger}$ \\
\hline Montenegro & $0.96(0.60-1.56)$ & $0.89(0.49-1.60)$ \\
\hline Serbia & $1.93(1.12-3.33)$ & $1.56(0.79-3.10)$ \\
\hline \multicolumn{3}{|l|}{$\begin{array}{l}\text { Region of the } \\
\text { Americas }\end{array}$} \\
\hline Belize & $1.67(0.88-3.16)$ & $13.95(1.91-102.02)^{\dagger}$ \\
\hline Panama & $3.76(1.88-7.53)$ & $5.14(2.37-11.14)^{\dagger}$ \\
\hline \multicolumn{3}{|l|}{ South-East Asia } \\
\hline Myanmar & $3.76(1.88-7.53)$ & $4.13(0.86-19.94)$ \\
\hline Sri Lanka & $2.30(0.98-5.40)$ & $2.29(0.75-6.98)$ \\
\hline \multicolumn{3}{|l|}{ Western Pacific } \\
\hline Mongolia & $2.70(1.11-6.52)$ & $1.52(1.19-1.94)^{\dagger}$ \\
\hline Philippines & $1.02(0.95-1.08)$ & $1.01(0.91-1.12)$ \\
\hline
\end{tabular}

Note: Referent category=exposure to $\leq 1$ source of pro-tobacco

advertisements; $C O R$, crude odds ratios; $a O R$, adjusted odds ratios; $\mathrm{Cl}$, confidence interval; FYR Macedonia, the Former Yugoslav Republic of Macedonia.

*Adjusted for age and sex.

t Statistically significant at the $\mathrm{P}<0.05$ level

reduce youth exposure to pro-tobacco advertisements from all sources.

The WHO and several public health and health professional organization have recommended classifying movies with tobacco incidents as $\mathrm{R}$ rating, with the exception of those that portray a historical figure who smoked and those that portray the negative effects of tobacco use [18-20]. Adopting this policy may further reduce exposure to tobacco incidents in youth-rated movies. This may however not completely eliminate youth exposure to smoking incidents in movies because some youths may still view some R-rated movies [19]. Hence, other interventions that have been recommended include requiring strong anti-tobacco advertisements preceding movies that depict smoking, not allowing cigarette brand displays in movies, and requiring producers of such movies to certify that no persons or company associated with the production received any consideration for that depiction $[18,20]$.

While the WHO Framework Convention on Tobacco Control encourages the banning of all tobacco product advertising, specific national legislation is required [13]. Very few countries have implemented $100 \%$ ban on tobacco advertisements without exemptions. Point-ofpurchase advertising is the commonest exemption in countries that have implemented policies restricting tobacco advertisements [21], as is the case in countries such as Serbia, South Africa, Philippines, Pakistan, the Former Yugoslav Republic of Macedonia, Costa Rica, Cameroon and Algeria [6]. In Indonesia, even though very few restrictions exist on cigarette advertisements, local manufacturers still concentrate their efforts on point-of-purchase advertising as it is considered a low investment, high impact, and 24-hour advertisement [6].

Notably, some countries have implemented partial restrictions on point-of-purchase advertisements, such as those delimiting the distance of outlets with such advertisements from a school, or restricting where such point-of-purchase advertisements may be placed within outlets. For example, legislation in South Africa restricts point-of-sale displays to within one meter of the cash register [6]. The WHO however favors complete restrictions [13] which involves putting all tobacco products out of view, as well as complete removal of other non-tobacco items (including signs, decals, mouse pads, till covers) containing tobacco product brand name, logo, motto, selling message, or recognizable color/pattern of colors.

Stronger enforcement of policies restricting tobacco advertisements in youth-oriented magazines as well as in newspapers may help reduce the prevalence of exposure to pro-tobacco advertisements through these media. Granted, this may not completely eliminate youth exposure to pro-tobacco advertisements in newspapers/magazines since some youths may still be exposed to such advertisements in international newspapers/magazines in print form or over the internet. However, if such restrictions are coupled with population based strategies that denormalize tobacco use, such as hard-hitting 
media campaigns that warn about the dangers of tobacco use, this may help prevent smoking initiation among never smokers, and prevent relapse among youths that have quit smoking. In addition, this may encourage youths that currently smoke to quit [22].

In 2007, the Nigerian federal government and a few state governments initiated lawsuits against three major tobacco companies: British American Tobacco, Phillip Morris and International Tobacco, for allegedly targeting under-aged minors with tobacco products [23]. While there has been no resolution to these cases to date, this development is however interesting as this is the first litigation between a developing country and the tobacco industry. How this case will affect tobacco promotional activities in Nigeria and other LMICs remains to be seen. Nonetheless, considering the heavy toll that smoking and secondhand smoke-attributable diseases place on already stretched health resources in developing countries [24-26], it is important for national governments in LMICs to invest in evidence-based strategies that will reduce tobacco use among youths, and avert excess death and disease.

\section{Strengths and limitations}

This study compared exposure to pro-tobacco advertisements among adolescents in several LMICs. Nationally representative data from students aged 13-15 years old from 20 LMICs in all six WHO geographic regions was assessed for the period 2007-2008. However, these findings are subject to at least five limitations. First, exposure to pro-tobacco advertisements was self-reported, and may have been subject to recall bias. Second, these data apply only to adolescents that attend school. However, in most of the countries assessed, the vast majority of adolescents aged 13-15 years are enrolled in traditional school, and thus the results may be representative of most of the students in this age bracket in the respective countries [15]. Third, the heterogeneity in school grading/class systems in the different countries made it impossible to meaningfully break down the analysis by school or grade level. Fourth, these findings may not be generalizable to respective regions as there were limited countries for which data was available. Finally, more recent data was not available because of the 4-year cycle of the GYTS.

\section{Conclusion}

This study demonstrated that the overall prevalence of exposure to pro-tobacco advertisements from different sources including movies, television programs, newspapers/magazines and outdoor events was high among adolescents aged 13-15 years in all 20 LMICs surveyed. In addition, exposure to multiple sources of pro-tobacco advertisements was associated with smoking behavior among adolescents in several LMICs. Enhanced and sustained national efforts in LMICs are needed to reduce exposure to all forms of tobacco advertising and promotional activities.

\section{Competing interests}

The authors have no competing interests to report.

\section{Authors' contributions}

ITA initiated the study design, analyzed the data and prepared the initial draft of the manuscript. $\mathrm{AOA}, \mathrm{AOA}$, and SOA critically reviewed and revised the manuscript. All authors read and approved the final manuscript.

\section{Acknowledgment}

Dr. Israel Agaku initiated the reported research while affiliated with the Africa Tobacco Control Regional Initiative. He is currently affiliated with the Centers for Disease Control and Prevention's Office on Smoking and Health. The research in this report was completed and submitted outside of the official duties of his current position and does not reflect the official policies or positions of the Centers for Disease Control and Prevention.

\section{Author details}

'Africa Tobacco Control Regional Initiative, Plot 397B, George Crescent, Agbalajobi Estate, Off Wempco Road, Lagos, Ogba-Ikeja, Nigeria. Department of Oral Pathology, College of Medicine, University of Ibadan, Ibadan, Nigeria. ${ }^{3}$ Department of Oral and Maxillofacial Surgery, University of Ibadan, Ibadan, Nigeria.

Received: 15 May 2013 Accepted: 21 May 2013

Published: 23 May 2013

\section{References}

1. World Health Organization: Confronting the epidemic: a global agenda for tobacco control research. 1999. Available at http://www.who.int/tobacco/ research/en/print.html. Accessed May 12, 2013

2. Euromonitor International: Tobacco: middle east and Africa regional overview, Retrieved from euromonitor passport GMID database. 2011. Accessed May 12, 2013.

3. Euromonitor International: Tobacco in Asia pacific: driver of the global market -will It remain the tobacco market powerhouse? Retrieved from Euromonitor passport GMID database. 2010. Accessed May 12, 2013.

4. Euromonitor International: Still a Smoker's paradise: a snapshot of the lighting-up culture in the Middle East and Africa, Retrieved from Euromonitor passport GMID database. 2012. Accessed May 12, 2013.

5. Euromonitor International: The future of tobacco, Retrieved from Euromonitor passport GMID database. 2011. Accessed May 12, 2013.

6. Euromonitor International: Country reports, Retrieved from Euromonitor passport GMID database. 2011. Accessed May 12, 2013.

7. Agaku I, Akinyele A, Oluwafemi A: Tobacco control in Nigeria- policy recommendations. Tob Induc Dis 2012, 10. 8-9625-10-8.

8. Agaku T, Alpert HR, Vardavas Cl, Adisa AO, Connolly GN: Use of smokeless tobacco and cigarettes among Nigerian youths: implications for tobacco control policies in Africa. Journal of Substance Use:1-6. 0 0:0.

9. U.S Department of Health and Human Services: Preventing tobacco Use among youth and young adults: a report of the surgeon general. Atlanta, GA: U.S Department of Health and Human Services, Centers for Disease Control and Prevention, National Center for Chronic Disease Prevention and Health Promotion, Office on Smoking and Health; 2012.

10. Hafez N, Ling PM: How Philip Morris built Marlboro into a global brand for young adults: implications for international tobacco control. Tob Control 2005, 14:262-271.

11. Friedman LC: Tobacco industry use of corporate social responsibility tactics as a sword and a shield on secondhand smoke issues. J Law Med Ethics 2009, 37:819-827.

12. FTC: Federal trade commission cigarette report for 2009 and 2010. 2013. Available at http://www.ftc.gov/os/2012/09/120921cigarettereport.pdf. Accessed February 21, 2013.

13. World Health Organization: WHO framework convention on tobacco control. 2012. Accessed September 23, 2012. 
14. Madkour AS, Ledford EC, Andersen L, Johnson CC: Tobacco advertising/ promotions and adolescents' smoking risk in Northern Africa. Tob Control 2013. Epub ahead of print.

15. Warren CW, Jones NR, Eriksen MP, Asma S, Global Tobacco Surveillance System (GTSS) collaborative group: Patterns of global tobacco use in young people and implications for future chronic disease burden in adults. Lancet 2006, 367:749-753.

16. The World Bank: Country and lending groups. 2013. Available at http://data worldbank.org/about/country-classifications/country-and-lending-groups. Accessed May 12, 2013.

17. World Health Organization: Parties to the WHO framework convention on tobacco control. 2013. Available at http://www.who.int/fctc/ signatories_parties/en/. Accessed May 12, 2013.

18. World Health Organization: Smoke-free movies: from evidence to action. Geneva, Switzerland: World Health Organization; 2009. Available at http:// www.who.int/tobacco/smoke_free_movies/en. Accessed May 12, 2013

19. Sargent JD, Tanski SE, Gibson J: Exposure to movie smoking among US adolescents aged 10 to 14 years: a population estimate. Pediatrics 2007, 119:e1167-e1176.

20. Centers for Disease Control and Prevention (CDC): Smoking in topgrossing movies--United States. MMWR Morb Mortal Wkly Rep 2010, 60:910-913.

21. Euromonitor International: Global tobacco: will legislation kill the industry? 2009. Retrieved from Euromonitor Passport GMID database. Accessed May 12, 2013

22. World Health Organization: WHO report on the global tobacco epidemic, 2008the MPOWER package. 2008. Available at http://www.who.int/tobacco/ mpower/mpower_report full_2008.pdf Accessed September 23, 2012.

23. BBC News: Nigeria delays \$44bn smoking case. 2008. Available at http://news. bbc.co.uk/2/hi/africa/7186719.stm. Accessed May 12, 2013.

24. Nsimba SE, Sussman S: Tobacco advertisements and promotion industry on smoking in Tanzania: a review of negative public health implications for current and future generations. Tob Induc Dis 2006, 3:41.

25. Singh PN, Yel D, Kheam T, Hurd G, Job JS: Cigarette smoking and tuberculosis in Cambodia: findings from a national sample. Tob Induc Dis 2013, 11:8.

26. Charoenca N, Kungskulniti N, Tipayamongkholgul M, Sujirarat D, Lohchindarat S, Mock J, Hamann SL: Determining the burden of secondhand smoke exposure on the respiratory health of Thai children. Tob Induc Dis 2013, 11:7.

doi:10.1186/1617-9625-11-11

Cite this article as: Agaku et al:: A cross-country comparison of the prevalence of exposure to tobacco advertisements among adolescents aged 13-15 years in 20 low and middle income countries. Tobacco Induced Diseases 2013 11:11.

\section{Submit your next manuscript to BioMed Central and take full advantage of:}

- Convenient online submission

- Thorough peer review

- No space constraints or color figure charges

- Immediate publication on acceptance

- Inclusion in PubMed, CAS, Scopus and Google Scholar

- Research which is freely available for redistribution 